\section{Praxisnahe Vermittlung der Schwindeldiagnostik}

D as Buch vermittelt die Grundlagen der Diagnostik von Gleichgewichtsstörungen unter besonderer Berücksichtigung der thermischen Videonystagmografie. Es werden zunächst die anatomischen und physiologischen Grundlagen des Vestibularsystems sowie die Anamnese und klinischen Untersuchungen und anschließend die apparativen diagnostischen Möglichkeiten zusamhalten. HNO-Ärzte in Klinik und Praxis. mengefasst. Die Darstellung des Vorgehens bei der Diagnostik der einzelnen Krankheitsbilder ist sehr praxisnah ge-

Das Buch beseitigt viele Unsicherheiten in der Diagnose der zugrunde liegenden Ursachen von Gleichgewichtsstörungen, die es nach wie vor gibt. Für

$g z$

\section{Verbessertes Nachschlagewerk}

$B^{c}$ ereits nach fünf Jahren legen die Herausgeber der „Bildgebung $\mathrm{HNO}-$ Heilkunde" eine Neuauflage vor, diesmal in Atlasform, was dem Charakter des Buches als Nachschlagewerk entgegenkommt. Einzelne Kapitel wurden um neu hinzugekommene Verfahren wie beispielsweise die DVT, die gerade im Niedergelassenenbereich in der Diagnostik chronisch entzündlicher Nasennebenhöhlenerkrankungen zunehmend an Bedeutung gewinnt, sowie "dynamische" Bildgebungsverfahren wie die Diffusions- und PET-Bildgebung erweitert.
Eine weitere Verbesserung wurde durch die Integration einiger Patientenbilder erreicht; trotzdem würde man sich hier für die Zukunft mehr wünschen! Korrespondierende klinische und bildgebende Befunde erleichtern das Verständnis und die Kommunikation zwischen Radiologen und zuweisendem Arzt, insbesondere, wenn es sich um Bildmaterial handelt, das dem "normalen" Auge sonst verborgen bleibt, also vor allem endoskopische Befunde.

Auch die nun erschienene, überarbeitete und erweiterte Neuauflage ist ansons-

\title{
Gut lesbares Spezialwissen
}

$\mathrm{m}$ Kindesalter ist eine Audiometrie ohne objektive Messverfahren heute kaum mehr denkbar. Die Autoren sind bekannt für ihre Erfahrung mit der Impedanzaudiometrie und vor allem mit otoakustischen Emissionen und der Ableitung akustisch evozierter Potentiale.

In diesem Buch steht die klinische Anwendung dieser Hörprüfverfahren im Vordergrund, aber auch eigene Ergebnisse werden evaluiert und technische Weiterentwicklungen erläutert.

Sehr ausführlich beschrieben ist das Kapitel „Akustisch evozierte Potentiale“, welches frühe (FAEP und BERA), mittlere (MAEP), späte (SAEP) akustisch evozierte Potentiale umfasst sowie ereigniskorrelierte Potentiale (ERP) und stationäre Potentiale des auditorischen Systems (ASSR) und die Elektrocholeografie berücksichtigt. Auch das Kapitel über Cochlea-Implantation erläutert ausführ- lich präoperative Diagnostik und postoperative Erfolgskontrollen.

Das Werk ist umfassend, übersichtlich und gut lesbar. Es ist eines der wenigen Werke in deutscher Sprache, das die objektive Audiometrie komplett abhandelt. Es stellt sich allerdings die Frage, welchem Leserkreis es zu empfehlen ist. Für einen Arzt in Ausbildung ist es zu speziell und für einen niedergelassenen oder klinisch tätigen Arzt bieten die Kapitel "Anatomische und physiologische Grundlagen und Pathophysiologie" ebenso wenig Neues wie die „Klinik der kindlichen Hörstörungen oder Screening und Früherkennung". Ein niedergelassener HNO-Arzt oder Kinderarzt wird eher das übersichtliche OAEHandbuch von Hoth und Neumann lesen. Der geringe Kreis von Spezialisten, der sich in großen Kliniken mit den verschiedenen Arten von akustisch evozier-
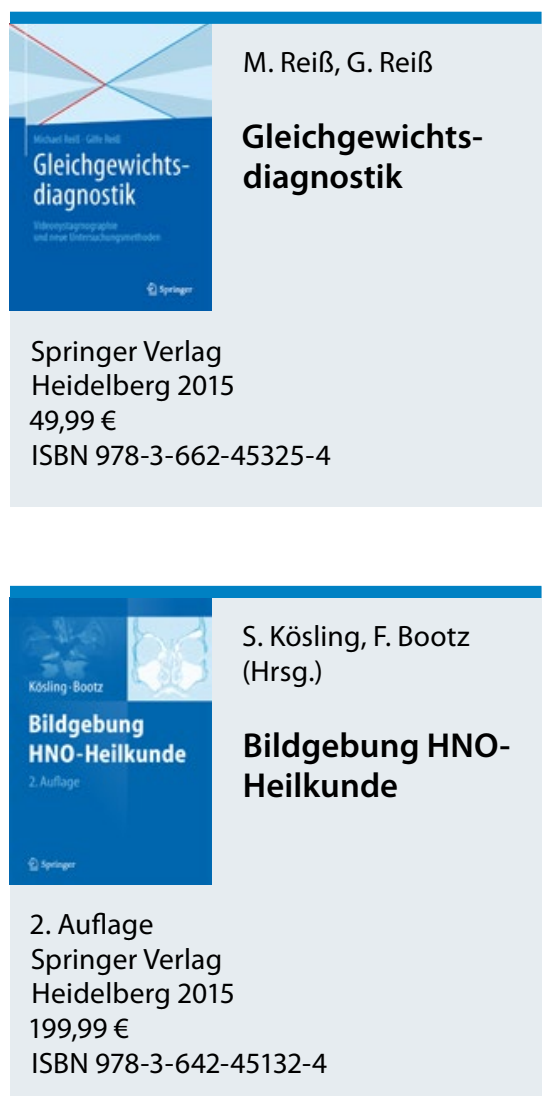

ten als Nachschlagewerk vorbehaltlos zu empfehlen. Prof.Dr. med. Gerhard Grevers

ten Potentialen beschäftigt und auch Voruntersuchungen vor CI durchführt, wird sich in Veröffentlichungen belesen, welche mehr technische Einzelheiten beinhalten. Trotz eines vernünftigen Preises von $80,00 €$ dürfte das übersichtliche Werk deshalb voraussichtlich nur für einen überschaubaren Leserkreis bestimmt sein.

Prof. Dr. Karin Schorn

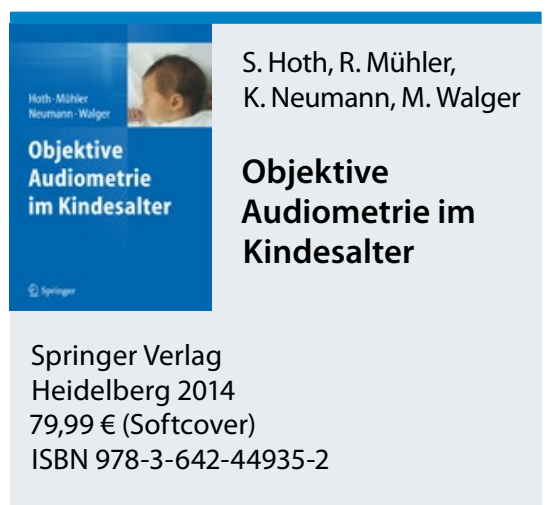

